\title{
50th anniversary of computed tomography: past and future applications in clinical neuroscience
}

\author{
William P. Dillon $\odot *$ \\ University of California, San Francisco, Department of Radiology and Biomedical Imaging, \\ San Francisco, California, United States
}

\begin{abstract}
Purpose: It has been 50 years since computed tomography was introduced to the worldwide neurologic medical and surgical community. In that time, tremendous advances in computer software and hardware, as well as creative changes in computerized tomographic (CT) hardware and tube technology, have dramatically improved the temporal and spatial resolution of CT. In this paper, I address what I feel are some of the most important impacts of CT in the field of clinical neuroscience over the last 50 years, as well as potential applications of CT that are on the horizon.

Approach: I have recounted from literature, colleagues, and personal recollection the historical impact of CT on neuroradiology practice and what appear to be near-term future applications.

Conclusions: Therapeutic applications beyond diagnosis, such as image-guided procedures, radiation, and surgical planning, and development of the field of theranostics have emerged and further increased the need for faster and more precise CT imaging. The integration of machine learning into the acquisition chain and radiologist tool kit has great implications for standardization, analysis, and diagnosis worldwide.
\end{abstract}

(C) 2021 Society of Photo-Optical Instrumentation Engineers (SPIE) [DOI: 10.1117/1.JMI.8.5.052112]

Keywords: brain; computed tomography; neuroradiology; spine; technology.

Paper 21170SSRR received Jun. 30, 2021; accepted for publication Sep. 27, 2021; published online Oct. 18, 2021.

\section{Introduction}

It has been 50 years since the first generation of computed tomography was introduced to the worldwide neurologic medical and surgical community. In 1971, the first clinical head computerized tomographic (CT) scan was performed at Atkinson Morley's Hospital in London on a patient with a suspected frontal lobe tumor (Fig. 1). The surgeon confirmed that the lesion looked just like the picture. As a neuroradiology fellow at UCSF, my mentor, the late Dr. Hans Newton, recounted to me his first glimpse of a brain CT. He was visiting Dr. James Bull at Queen's Square in London. Dr. Bull asked Hans whether he would like to see the ventricles of a brain without using pneumoencephalography, the standard at the time. Hans was obviously intrigued as this was not possible without introducing air into the subarachnoid space. Bull then took Hans to see some of the first images of the new EMI scanner. Amazed, Hans flew home and convinced the chairmen of neurology and neurosurgical surgery at UCSF to accompany him to the office of the chief administrator of UCSF, Bill Kerr. Kerr bought, sight unseen, one of the first scanners in the US, and UCSF became, along with Mayo clinic and MGH in Boston, one of the first medical centers in the US with this new technology. The scanner was installed in 1974 at UCSF under the direction of Dr. David Norman, where it quickly became an essential tool for the diagnosis of neurologic disorders.

The initial EMI scanner, for which Sir Godfrey Hounsfield and Allen Cormack were awarded the 1979 Nobel Prize in Physiology or Medicine, produced two adjacent 10-mm slices in $5.5 \mathrm{~min}$, and required about the same time necessary for reconstruction (Fig. 2). Therefore, a complete

*Address all correspondence to William P. Dillon, William. Dillon@ucsf.edu 


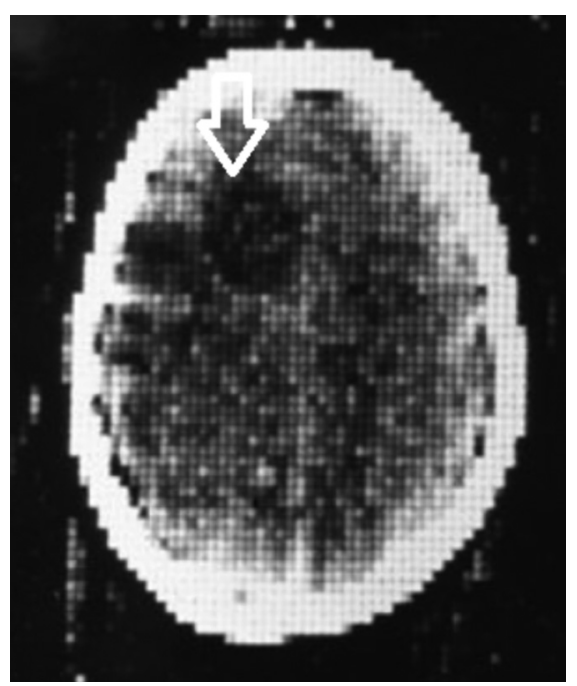

Fig. 1 The first CT scan in London, circa 1971. Right frontal low density tumor (arrow).

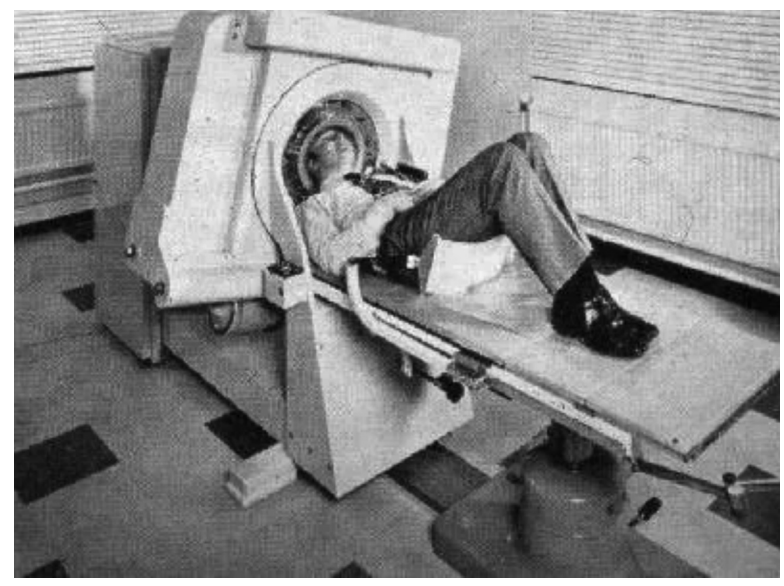

Fig. 2 EMI scanner circa 1971.

scan of 10 slices with a resolution of $80 \mathrm{~mm} \times 80 \mathrm{~mm}$ took about $30 \mathrm{~min}$ to acquire, $30 \mathrm{~min}$ to process, and a few additional minutes for filming on a Polaroid. As a resident at the University of Utah, one of our duties was filming the Polaroid images for the read out. Whole-body CT was introduced in the mid to late 1970s, and over the ensuing decades, improvements in hardware, computer power, and software dramatically improved the speed and resolution of CT, which now routinely acquires hundreds of 0.5 - to 0.625 -mm thick slices at $1024 \times 1024$ matrix size in seconds. Many physicians consider CT one of the most important advances in medicine in the 20th century. Its impact on the diagnosis and treatment of millions of patients with disorders of the brain, spinal cord and spine, and head and neck cannot be underestimated.

These 50 years of CT progress, including the development of helical CT, CT angiography, CT perfusion, dual-energy CT, and the combination of CT with positron-emitting tomography and single-photon emitting tomography, and its use in precise image-guided therapy, has been a golden era in neuroradiology. In this article, I will address what I consider nine of the most important impacts of CT in neuroradiology. What the next 50 years will bring to this technology will be of great interest. The integration of artificial intelligence (AI) into the mechanics of CT acquisition has already started, paving the way for reduced radiation exposure, noise reduction, and an even more detailed portrayal of anatomy and pathology. As machine learning improves, we expect to see it augment the diagnostic capabilities of radiologists, improving care, speed, and accuracy of diagnosis. Quantification of brain lesions, combined with genetic and radiomic information may improve diagnosis and prognosis. Perhaps even more important may be the 
impact of machine learning and $\mathrm{AI}$ in developing countries, where fewer radiologists exist and the potential for computerized diagnosis may improve diagnosis and treatment for millions of people worldwide.

\section{Infections of the Central Nervous System}

One of the most important impacts of CT has been in the diagnosis and monitoring of CNS infections. Early papers from Queen's Square ${ }^{1}$ and $\mathrm{MGH}^{2}$ brought to the attention of the neuroradiology community the appearance of intracranial infections on CT. Conditions such as cerebral abscess, subdural and epidural empyema are potentially morbid conditions if left untreated (Fig. 3). In 1977, as a medical student, the impact CT would have on the diagnosis and treatment of infectious diseases became personal for me. During my internal medicine rotation as a thirdyear student, a dear friend and classmate was admitted to our service with acute expressive aphasia. He had been suffering from sinusitis and allergies for the proceeding week or so. A plain film showed opacified frontal sinuses and an intracranial infection was suspected. I accompanied him down to the EMI scanner several times over the course of several days and watched as a subtle midline shift was eventually detected by the neuroradiologist. Drainage of the small subdural empyema and proper antibiotics allowed my colleague to continue his training and pursue his dreams, a family, and a brilliant career. In 1978, Rosenblum et al. reported that no deaths occurred among 20 consecutive patients with intraparenchymal brain abscesses treated at the University of California, San Francisco, since CT brain scanning became a routine diagnostic procedure. This was compared to 18 cases treated before the availability of CT in whom the mortality rate was $44 \%$ overall, and $36 \%$ for all operated patients treated. ${ }^{3}$ The use of intravenous

(a)

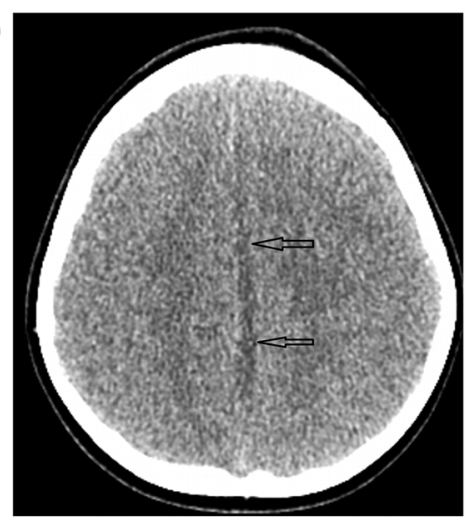

(b)

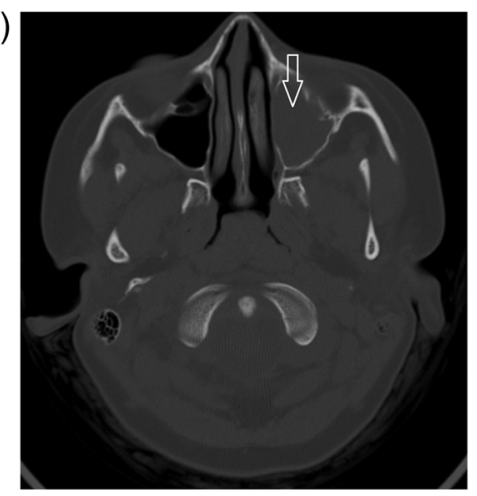

(c)

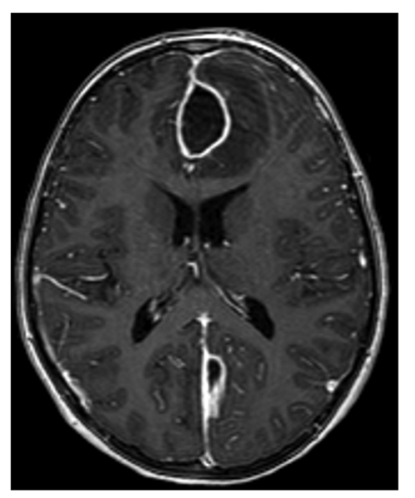

Fig. 3 Subdural empyema (pus in the midline subdural compartment) in a child secondary to sinusitis. (a) Non-contrast CT scan demonstrating interhemispheric collection of subdural empyema (pus) (black arrows) resulting from left maxillary sinusitis (b) CT of paranasal sinuses demonstrates opacification of the left maxillary sinus, consistent with sinusitis (white arrow). (c) Post-contrast T1 weighted MR image shows extensive abnormal enhancement surrounding interhemispheric subdural empyema. 
contrast during $\mathrm{CT}$ imaging followed rapidly and improved the diagnosis of several disease entities, especially cerebral abscesses, meningitis, and empyema. ${ }^{4}$

The introduction of whole-body CT scanners permitted views of the chest, abdomen, and the spine and spinal cord. The CT appearance of the spine was reported by several authors including Isherwood and Pullan. ${ }^{5}$ Brant-Zawadzki et al. ${ }^{6}$ also contributed their experience in CT of spine infections. While now MR provides more soft tissue detail than CT for the diagnosis of suspected intracranial and spinal infections, the rapidity of acquisition, resolution, and complementary osseous anatomic detail of CT remains major advantages, especially in very sick and uncomfortable patients. Today, total spine CT can be performed in seconds, and provides invaluable analysis of disc, facets, and vertebral bodies as well as the spinal cord and roots in the setting of suspected osteomyelitis, as well as providing image guidance for biopsies and aspirations. ${ }^{7} \mathrm{MR}$ imaging is complementary to $\mathrm{CT}$ and provides detailed information regarding disc space infections, paravertebral abscesses as well as spinal cord involvement. Nonetheless, CT still remains a critical imaging technique for the quick evaluation of spinal infections.

The year 2021 also marks the 40th anniversary of the beginning of the HIV/AIDs epidemic. San Francisco, where I work and live, was at the center of this scourge, and our hospitals were leaders in the science of HIV and the care of AIDs patients. CT, and later MR, played a pivotal role (and unfortunately still do) in the care of patients with HIV and AIDs by detecting lesions (many of which were infections), monitoring disease, and guiding precision neurosurgery when needed.

\section{Hemorrhage and Vascular Lesions}

Early in the CT experience, it became clear that intracranial hemorrhage was easily visible. ${ }^{8,9}$ Once hemorrhage begins to clot, which occurs in a matter of minutes, and the fibrin protein clot expels plasma, it appears dense on CT relative to surrounding brain. As the hematoma is gradually broken down by the body over a period of days to weeks, its density on CT resolves and may appear similar or lower in density to the normal brain. Prior to CT, the diagnosis of aneurysmal subarachnoid hemorrhage was made only with lumbar puncture and subsequent angiography, while intraparenchymal hemorrhage due to hypertension, trauma or underlying vascular malformations often were never diagnosed. Some lesions such as capillary telangiectasia, cavernous malformations, and hemorrhagic metastases were only recognized at autopsy. Thus, CT and later MR opened a window to pathology and diagnoses that neuroradiologists and our clinical colleagues only assumed were present based on clinical exam, surgical exploration, or pathology. Indeed, in my own career, many of the diagnoses we now consider routine in neuroradiology were only described in pathology textbooks prior to CT.

One of the key advancements in CT during the early 1980s was the introduction of automatic table incrementation. Prior to that, the table was advanced into the gantry manually, which was cumbersome at best. This advance, combined with improvements in tube and detector technology, allowed for faster scan times and multiplanar reformations of the digital data, which were a key to the development of CT angiography. As CT became faster, it was not long before it was used during intravenous contrast administration to assess vascular structures of the body, neck, and brain. Brant-Zawadzki and Jeffrey ${ }^{10}$ utilized CT with image reformations to screen the carotid bifurcation. This group was also one of the first to use CT reformations to image various anatomic regions of the body after trauma, including the maxillo-facial region and spine. ${ }^{11}$ In the 1990s, the introduction of slip ring technology and acquisition during fast table movement opened the field further as spiral or helical scanning was introduced. This, combined with more advanced computer reformations and surface rendering, sped the CT angiography revolution. ${ }^{12}$ The ensuing 30 years has seen CT angiography largely replace conventional angiography to become the primary diagnostic technique, along with MR angiography, to evaluate vessels throughout the body including the cervical and intracranial vessels. CTA is now used as a first-line tool in the evaluation of patients with acute stroke, aneurysmal subarachnoid hemorrhage, and suspected venous thrombosis. This has in turn led to more precise interventions and augmented the burgeoning field of interventional neuroradiology (Fig. 4). 
(a)

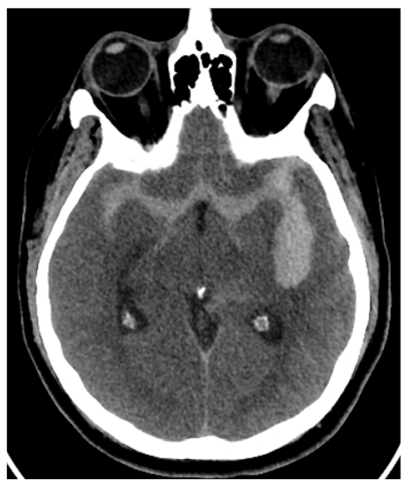

(b)

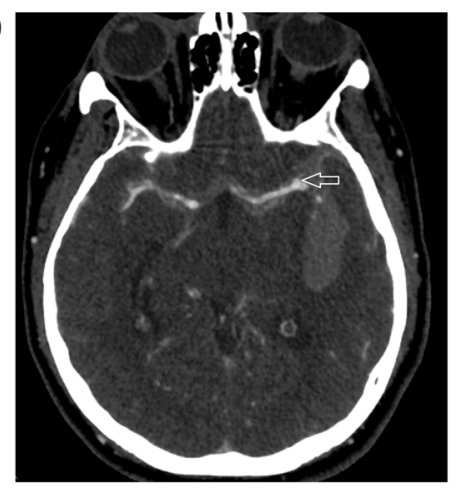

(c)

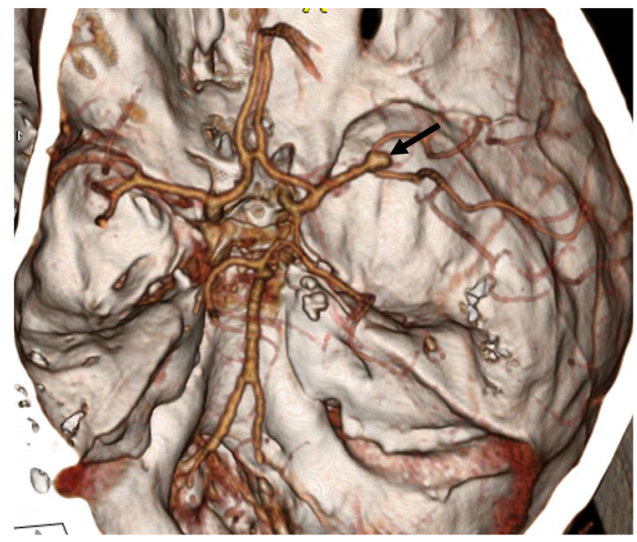

Fig. 4 Subarachnoid hemorrhage secondary to left middle cerebral artery (MCA) aneurysm. (a) Non-contrast CT scan shows high-density subarachnoid hemorrhage in the sylvian cisterns. (b) Single image from contrast-enhanced CT angiography series shows a focal outpouching of left MCA aneurysm (arrow). (c) 3D image of the left MCA aneurysm (arrow).

Helical CT scanning also permitted the development of CT perfusion techniques in the brain and body. This technique was an outgrowth of the use of nuclear medicine tracers to determine cerebral blood flow. In 1980, Axel described the theoretical possibility of using rapid CT acquisition to analyze CT perfusion characteristics, ${ }^{13,14}$ but the application required the development of helical CT to become robust and mainstream. Perfusion CT permitted calculation of cerebral blood volume, transit times, and cerebral blood flow, which have proved powerful additions in the assessment of acute ischemia, stroke and the characterization of brain tumors (Fig. 5). ${ }^{15,16}$

\section{Trauma}

Acute and chronic brain and spinal traumatic injury also unequivocally benefited from the development of $\mathrm{CT}^{9}{ }^{9,17}$ The detection of intracranial hemorrhage, fractures and their effect on brain and spinal cord had enormous clinical impact for patients in need of immediate surgical intervention. The relatively quick determination of subdural and epidural hematoma, the diagnosis of which previously required burr hole at the bedside, or cerebral angiographic detection of subtle shifts of blood vessels, was an enormous leap in clinical care of patients at trauma centers worldwide. As a neuroradiology fellow at UCSF in 1982, my first rotation was at the San Francisco General Hospital, the level 1 trauma facility for San Francisco County. SFGH had two CT scanners that operated around the clock, scanning patients with acute trauma, stroke and AIDs. Rapid reformations permitted detailed analysis of complicated fractures. ${ }^{18} \mathrm{CT}$ was also eventually incorporated into algorithms of care and prognostication for patients with major trauma. Indeed, major medical centers now incorporate CT and CTA in the emergency department for quick decision making. Currently, at the new Zuckerberg San Francisco General Hospital, trauma patients enter the ED immediately into a trauma room adjacent to the CT scanner, which is integrated into an angiography suite, where total body CT and angiography 
(a)

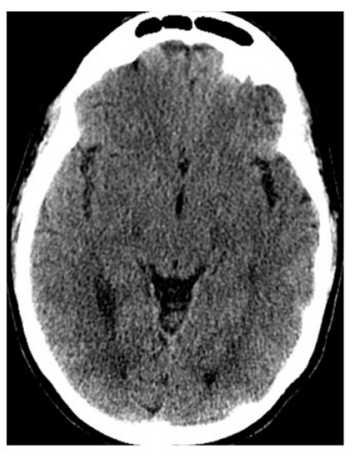

(d)

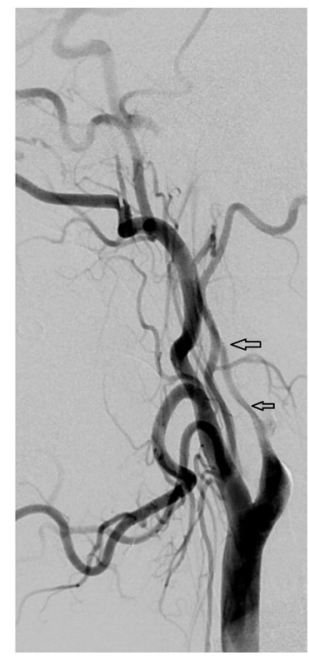

(b)

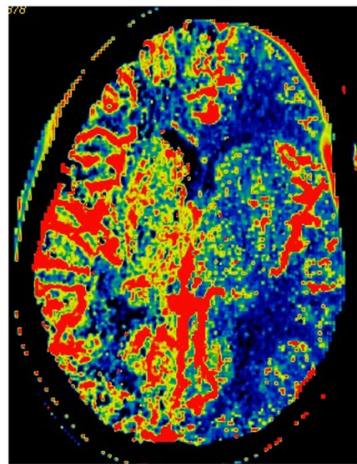

(e)

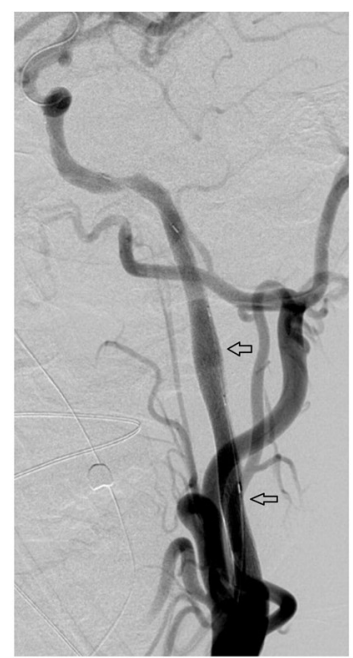

(c)

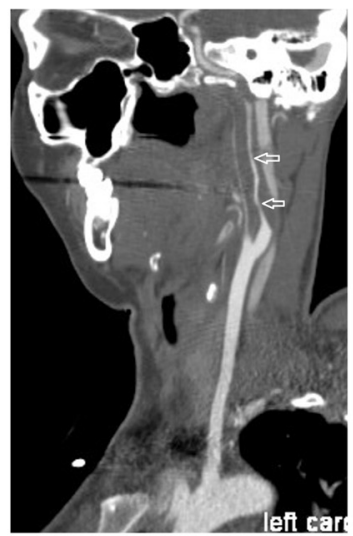

(f)

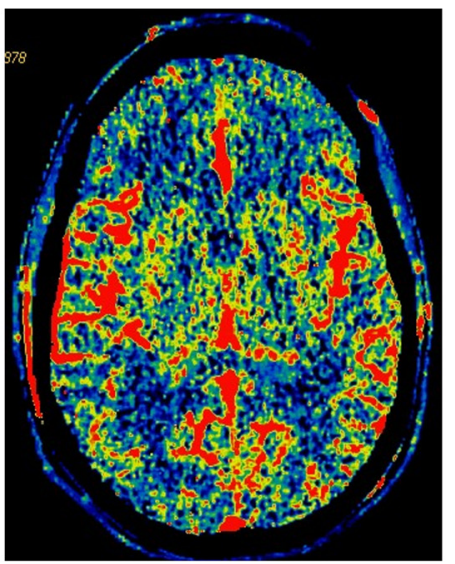

Fig. 5 Carotid dissection following chiropractic neck massage, with word finding difficulties prior to stent, with resolution post-stenting. (a) Normal non-contrast CT. (b) CT Perfusion blood flow image shows decreased relative cerebral blood flow in the left hemisphere (blue area). (c) Sagittal reformatted image from CT angiography series shows tapered narrowing of the left internal carotid artery, consistent with dissection (arrows). (d) Conventional angiography documents the dissection (arrows). (e) Conventional angiography after placement of carotid artery stent (arrows). (f) Post-stenting CT perfusion shows normalization of cerebral blood flow on the left with return to normal symmetric perfusion.

can be performed in literally a matter of minutes. It has been shown that immediate access to CT in the emergency department improves functional outcomes of those patients with blunt head trauma (Fig. 6). ${ }^{19}$

\section{Neuro Oncology}

The first CT scan ever performed was for a patient with a frontal lobe tumor. Since that 1971 scan, the impact of CT on brain tumor diagnosis has been enormous. While MR has largely replaced conventional CT for the diagnosis of many CNS disorders, including brain tumors, CT remains critical for patients presenting with acute deterioration, complications of prior therapy, as well as for those who cannot enter the magnet room or who are uncooperative (Fig. 7). Quick diagnosis of hemorrhage, hydrocephalus, or mass effect in patients with brain tumors can be lifesaving. Perhaps one of the most exciting developments in oncology, however, was the integration of CT with positron emission tomography (PET/CT). The additional value of $\mathrm{PET} / \mathrm{CT}$ and PET/MR in neuro oncology has yet to be fully realized as new radioisotope biomarkers, such as amino acid radiotracers, are being developed that promise to specifically differentiate tumor from treatment effects based on their specific uptake by metabolically active tumors. Quartuccio et al. reviewed the literature from 2015 through 2020 and showed that 
(a)

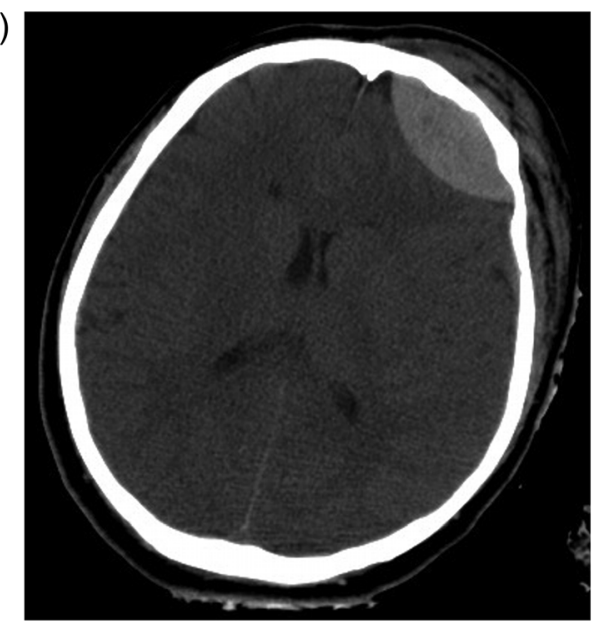

(b)

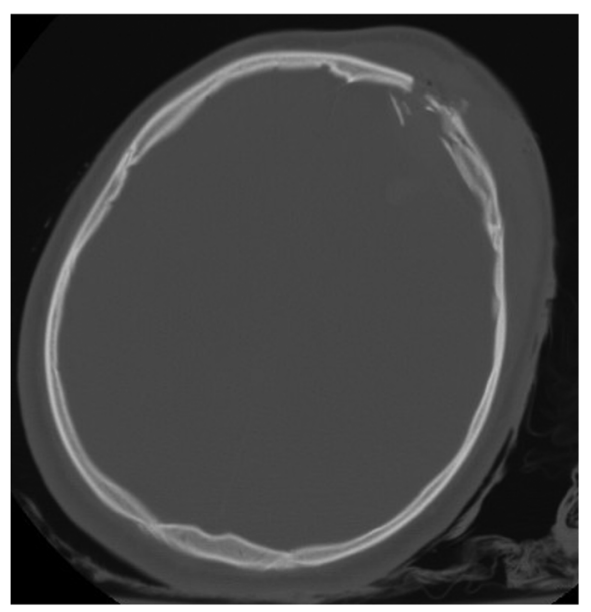

Fig. 6 Epidural Hematoma. (a) Non-contrast CT demonstrating left frontal extra axial lens-shaped epidural hematoma, with mass effect on frontal horns of the lateral ventricle. (b) Bone windows showing comminuted displaced fracture at the hematoma site.

PET/CT provides "different and complementary information to MR and may enhance performance in the whole management of gliomas. ${ }^{20}$ In addition to diagnosis and follow-up, CT and PET/CT play a critical role in radiation therapy planning ${ }^{21}$ and stereotactic surgery. Soon, so called neurotheranostics will combine cancer imaging with targeted molecular bullets that bind to cancer cells and provide imaging specificity as well as the potential for treating cancer cells with radioisotopes. ${ }^{22}$

\section{$6 \mathrm{Head} /$ Neck Diseases Including Hearing Loss}

My involvement in head and neck imaging occurred fortuitously during my residency at the University of Utah, where Dr. Anthony Mancuso was one of my professors. Tony had trained and worked closely with Dr. William Hanaffe at UCLA. Dr. Hanaffe had developed an international reputation as a leader in neuroradiology but then turned his creative genius to the field of otolaryngology, which at the time had little attention from radiology other than a few academic and private practice radiologists. The American Society of Head and Neck Radiology was in its infancy, founded in 1976 as an outgrowth of an annual meeting organized by Dr. Galdino Valvassori, and was largely devoted to plain film analysis of these structures. Drs. Hanafee and Mancuso embraced CT and applied it to the complex, beautiful anatomy of the head and neck, advancing the care of patients with hearing loss, sinonasal diseases, and head and neck cancer. CT rapidly became the gold standard for the detection and staging of cancers of the head and neck, and quickly replaced plain film tomography for the analysis of the temporal bone and skull base pathology (Fig. 8). ${ }^{23,24} \mathrm{CT}$ scanning remains a key technology for this 
(a)

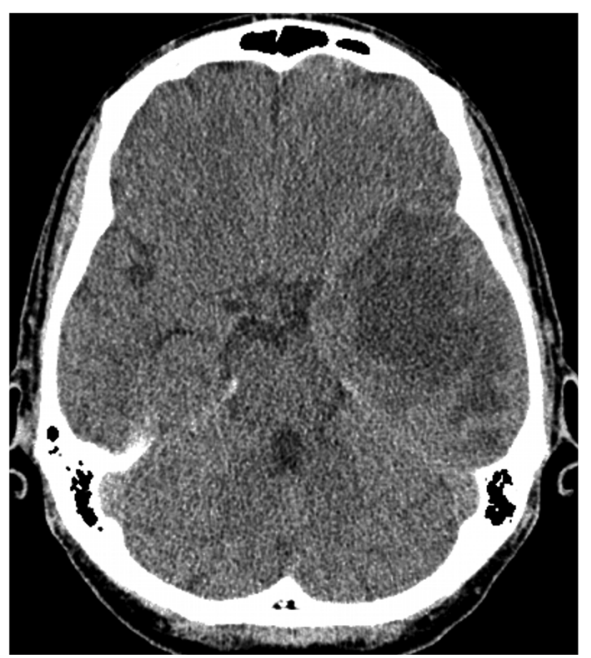

(b)

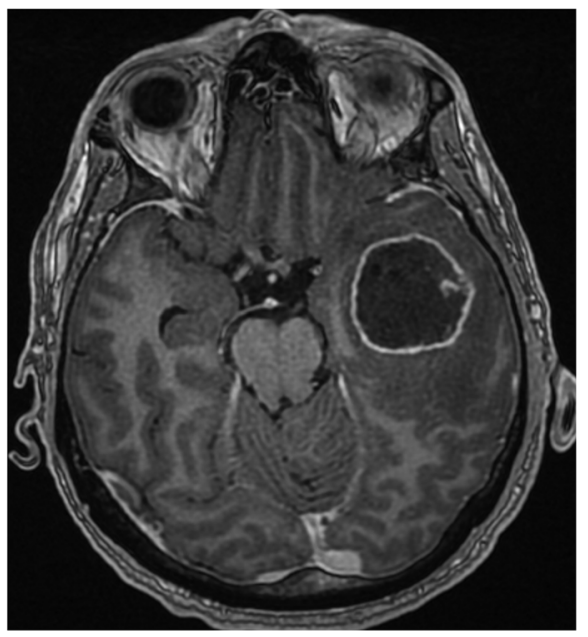

Fig. 7 Left temporal lobe glioblastoma. (a) Non-contrast CT shows left temporal lobe mass and edema. (b) T1 weighted gadolinium contrast enhanced MR scan demonstrates the mass shown in $5 \mathrm{~A}$ as a ring-enhancing left temporal lobe mass.

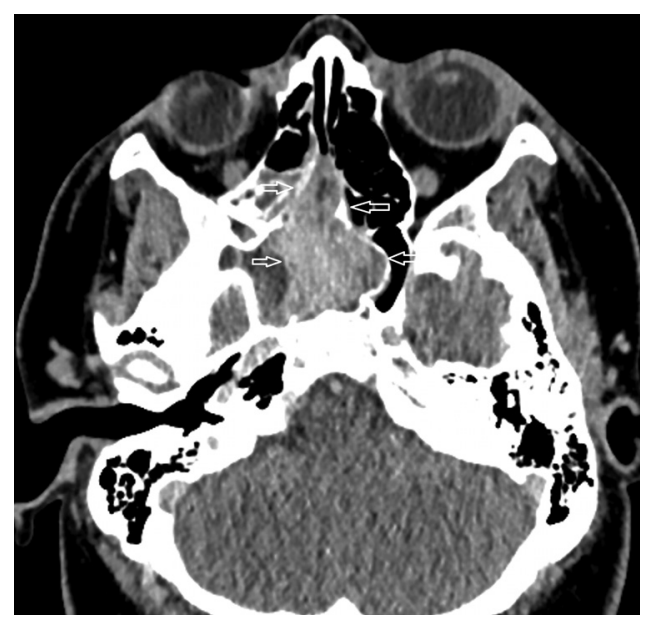

Fig. 8 Juvenile angiofibroma. Contrast-enhanced CT scan through the skull base and sphenoid sinus reveals an enhancing mass lesion centered in the sphenoid sinus extending into the posterior nares (arrows). 
anatomy, which, unlike the brain, moves with swallowing and breathing. Clever techniques were devised such as introducing subarachnoid air via lumbar puncture, so-called CT air cisternography, for evaluation of tumors of the vestibular nerve in the internal auditory canal and puffing the cheeks out with air while scanning patients with suspected oral cavity pathology. ${ }^{25,26}$ Dynamic CT scanning during a bolus of intravenous contrast is essential to differentiate vascular structures from lymph nodes, as well as providing differential contrast opacification of certain tumors such as parathyroid adenomas, juvenile angiofibromas and perineural spread of cancer. Coronal CT reformations also provide the otolaryngologist with a map for endoscopic sinus surgery, which revolutionized the care of patients with sinonasal pathology.

\section{Back Pain}

Back pain is the third leading cause of disability in the United States and accounts for a large percentage of health care expenditure. ${ }^{27}$ In a cohort study of nearly 2.5 million US patients diagnosed with low back or lower extremity pain between 2008 and 2015, only $1.2 \%$ received surgery, but this subset of patients accounted for $29.3 \%$ of total 12-month costs, largely due to imaging. ${ }^{28}$ Shortly after the introduction of the whole-body scanners, spine imaging with CT was implemented and used clinically. ${ }^{12,29}$ Di Chiro and Shellinger ${ }^{30}$ reported on the use of CT myelography and Drayer and colleagues reported on the use of CT following introduction of intrathecal contrast via lumbar puncture (CT cisternography) for assessment of intracranial cysts, CSF leaks, and hydrocephalus (Fig. 9). ${ }^{30}$ The impact of CT on the diagnosis of back and neck pain was an enormous advance in the care of such patients and transformed spinal surgery for acute and chronic spinal abnormalities, including congenital lesions, trauma, tumors, and degenerative diseases. MR is now the study of choice for patients with back pain as it shows disc and cord abnormalities much better than CT. But CT remains the mainstay for acute traumatic lesions, and is complementary to MR in patients with advanced degenerative disease and in those with failed back in whom metallic implants create artifacts on MR. The standard CT protocol now involves thin sections that can be reformatted in any plane. In addition, CT is now used extensively to guide spinal interventions such as epidural nerve blocks, blood patches, as well as for difficult lumbar punctures, dynamic myelograms for CSF leak syndromes and vertebral augmentation. The use of CT for image guided biopsy, injections and therapy has exploded in the past 10 years, with many facilities dedicating scanners for interventional use. Unfortunately, however, CT and now MR are also major drivers of health care expenditure, especially in patients with back and neck pain. Their immediate use in patients with acute pain, without neurologic deficits or immunocompromised states/ cancer, should be minimized as back pain often resolves within a few weeks with conservative management.

The combined use of PET with CT has also made an impact on the diagnosis and therapy of spinal pain. CT PET studies using fluorodeoxyglucose (18F-FDG) or sodium fluoride (NaF) ligands have been used to detect metastatic disease, but also to evaluate the source of back pain including facet arthritis. ${ }^{31-33}$

\section{Hydrocephalus and Disorders of CSF Pressure}

As Hans Newton witnessed in London, CT changed the ballgame for assessing the ventricular system. The immediate impact allowed the diagnosis of hydrocephalus as well as causes of obstruction. ${ }^{34}$ Obstruction of the CSF pathway may occur within the ventricular system-so called non-communicating hydrocephalus - as well as outside the ventricular system in the extra ventricular subarachnoid spaces, which is referred to as communicating hydrocephalus. Communicating hydrocephalus with normal pressure (NPH) was also recognized as a treatable clinical entity by Hakim and Adams. ${ }^{35} \mathrm{NPH}$ occurs secondary to trauma or subarachnoid hemorrhage or may be idiopathic without preexisting reasons for obstruction. The cause(s) of idiopathic NPH remain somewhat of a mystery even after all the years of CT and MR availability. Some features associated with NPH include enlarged ventricles out of proportion to sulcal size, enlarged size of sulci below versus near the vertex, and periventricular low density around enlarged ventricles. Imaging studies are useful in (1) excluding pathologic obstruction of 
(a)

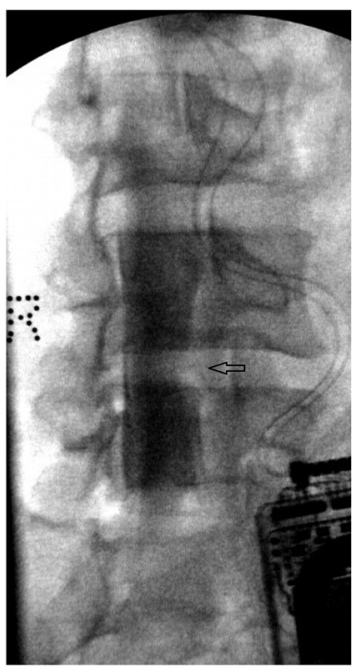

(b)

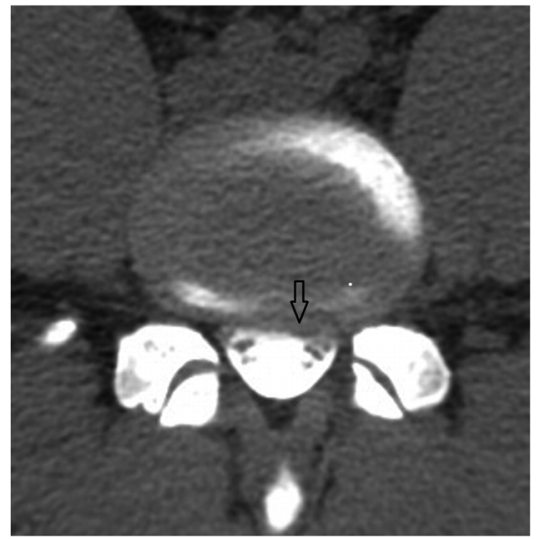

(c)

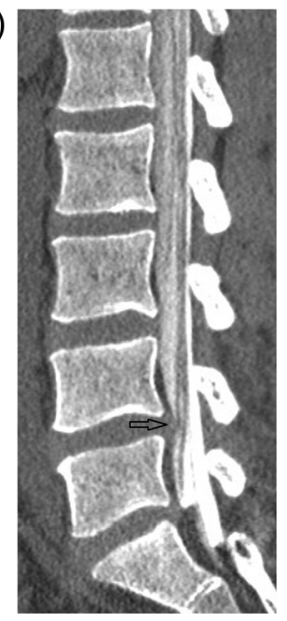

Fig. 9 Myelogram and CT myelogram in a patient with left-sided back pain and radiculopathy. (a) Conventional lumbar myelogram shows a left extra axial defect at L 4-5 (arrow) caused by a disc protrusion indenting the opacified thecal sac. (b) Axial CT post myelogram shows left central disc protrusion (arrow) indenting the left ventral opacified thecal sac. (c) Sagittal reformatted CT myelogram shows left central disc protrusion (arrow).

CSF pathways, (2) following patients after shunting, and (3) and raising the possibility of NPH in patients presenting with dementia and or gait abnormalities. Contrast cisternography was introduced by some authors to detect CSF pathway obstruction or resistance to CSF absorption. ${ }^{36}$

While we cannot yet directly measure pressure with CT, both high and low CSF pressure disorders may result in indirect features on CT. A change in ventricular size requires a difference in the trans mantle pressure between the subarachnoid and ventricular compartments. Some lesions, such as idiopathic intracranial hypertension (IIH) may not result in a difference in trans-mantal pressure and thus show no change in ventricular or sulcal size, despite quite elevated pressures. IIH is a syndrome typically in overweight women with increased CSF pressure who present with headache, pulsatile tinnitus and or visual symptoms. As the name implies, the cause(s) of IIH are obscure but may relate to increased resistance of intracranial venous return to the chest in obese patients. CT features of IIH are subtle and not definitive, but include dilated peri-optic CSF spaces, downward displacement of the pituitary gland by CSF (partially empty sella), and focal narrowing of the transverse sinuses.

Low CSF pressure syndromes may also manifest on CT and MR. Spontaneous intracranial hypotension (SIH) is a syndrome presenting typically with postural headaches, and variable features on imaging related to loss of CSF volume. These include sagging of the hindbrain structures, enlarged venous sinuses, dural enhancement, enlarged pituitary gland, subdural CSF or 
(a)

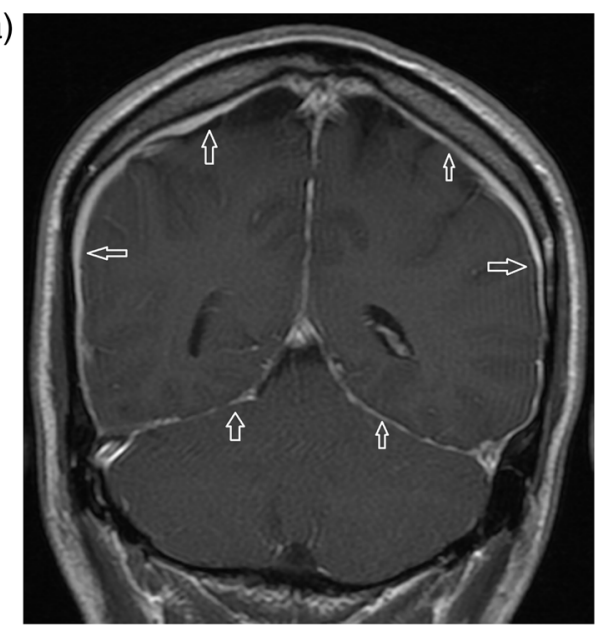

(b)

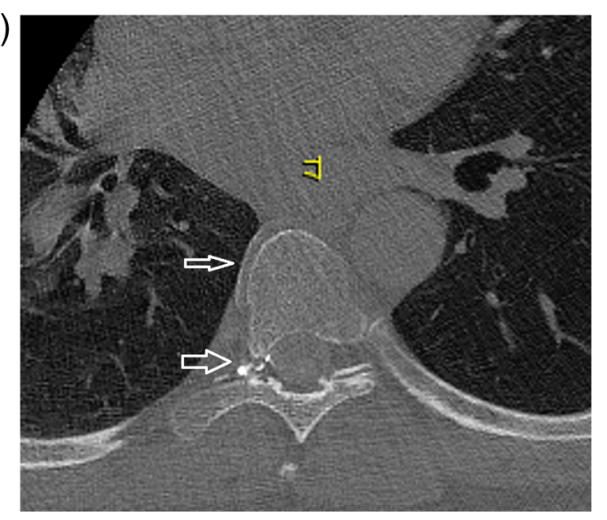

Fig. 10 Dynamic CT myelogram shows a CSF venous fistula causing spontaneous intracranial hypotension. (a) Contrast-enhanced MR scan of the brain demonstrates diffuse dural enhancement (arrows) in this patient presenting with postural headaches. This is typical of spontaneous intracranial hypotension (SIH). (b) Decubitus CT dynamic myelogram demonstrates opacification of a CSF-venous fistula in the thoracic spine. The arrows indicate the connection between a nerve root and a periradicular vein, resulting in low CSF volume and pressure-causing $\mathrm{SIH}$.

hematoma collections and others. ${ }^{37,38,39,40}$ The causes are numerous and include trans-dural herniation of disc or osteophyte, rupture of perineural diverticula, and CSF-venous fistula among others. Diagnosis and treatment of these lesions involves dynamic myelography, with CT or fluoroscopy, and CT-guided epidural blood and fibrin glue patching of leaks (Fig. 10). ${ }^{40,41}$

\section{Pediatric Lesions of the CNS}

The issue of radiation exposure is a significant issue in the use of $\mathrm{CT}$, especially in the pediatric population. CT represents $5 \%$ to $10 \%$ of all imaging procedures, but $40 \%$ to $70 \%$ of the collective medical radiation dose, and has been associated with increased risk of neoplasms. ${ }^{41,42}$ MR has thus largely replaced CT for this population. Nonetheless, CT was used frequently for many years prior to the advent and dissemination of MR imaging and is still used extensively in pediatric trauma. ${ }^{43,44} \mathrm{CT}$ opened the door to our better understanding of pediatric brain tumors, congenital malformations, hydrocephalus, vascular and traumatic lesions, including non-accidental trauma (NAT) (i.e., child abuse), acute stroke as well as congenital spinal anomalies. There is still a role for CT in some of these lesions in the emergency setting, but it is quite limited. Especially important was the impact of CT on the diagnosis of NAT. CT features more frequently seen in NAT include subdural hemorrhage adjacent to the midline falx (a fibrous leaf of the dura in the midline), subdural hemorrhage, large (non-acute) extra-axial fluid, and basal ganglia edema. ${ }^{45,46}$ 
(a)

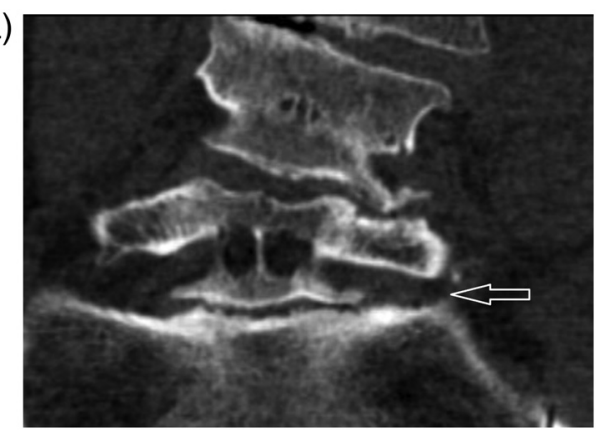

(b)

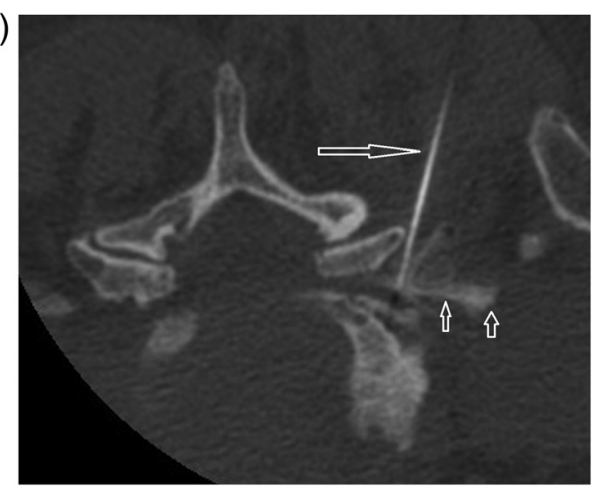

Fig. 11 CT guided nerve block. (a) Coronal reformatted CT image of the lumbar spine in a patient with left $L 5$ radiculopathy showing narrowing of the L5 foramen due to degenerative scoliosis (arrow). (b) +Axial prone CT guided nerve block. Needle (long arrow) is positioned through the narrowed opening injecting contrast-enhanced medication (short arrows) around the left L5 nerve root.

\section{Image-Guided Procedures}

Throughout this paper, we have mentioned the use of CT guidance for diagnostic and therapeutic interventions. This tool has increased precision diagnosis and therapy in all areas of the body. In our facility, a dedicated CT scanner for outpatient spine interventions has proven beneficial to our patients with back and neck pain, those with spontaneous intracranial hypotension (CSF leaks) in need of blood patches, patients in acute pain secondary to vertebral compression fractures (kyphoplasty), and those requiring a biopsy for suspected malignancy or infection of the spine (Fig. 11). CT (and MR) is also used by our surgeons and radiation therapist to plan surgery and intensity modulated radiation therapy, respectively. The dose needed for many of these interventions can be kept very low, and in many cases improved precision over fluoroscopic procedures is an added benefit. ${ }^{47,48}$ That said, the radiation dose with CT must be kept in mind as it is quite easy to increase dose over that of fluoroscopic-directed interventions. ${ }^{49}$

\section{Summary}

The 50 years since the first brain CT was performed in London has seen dramatic advances in medicine, computer hardware and software, and novel creativity of scientists and radiologists to bring about new diagnostic and therapeutic advances. What will the next 50 years bring in this technology? Clearly, over the next five years, we will see further integration of machine learning into the image acquisition and processing chain, and probably augmentation of diagnostic radiology interpretations. Machine learning may also spread this technology to a wider population in developing countries who now may have a CT but lack radiology expertise. The development of more specific radiotracers will continue to improve PET/CT and PET/ MR, augmenting the anatomic detail with functional information. As we write, new technology using solid-state CT scanners without moving parts are being developed for long-term space flights. Integration of 
CT scanners into ambulances is already occurring in many cities allowing faster diagnosis of acute stroke and hemorrhage while in the field. Portable CT scanners move about the hospital now for those patients who cannot be safely transported to the radiology department. There is no doubt CT will continue to play a key role in medicine and health care worldwide in the coming decades.

\section{Disclosures}

No conflicts of interest, financial or otherwise, are declared by the author.

\section{References}

1. J. W. D. Bull et al., "First results with an EMI scanner," Acta Radiol. Diagn. 16(347_suppl), 495-498 (1975).

2. P. F. New, K. R. Davis, and H. T. Ballantine Jr., "Computed tomography in cerebral abscess," Radiology 121(3 Pt. 1), 641-646 (1976).

3. M. L. Rosenblum et al., "Decreased mortality from brain abscesses since advent of computerized tomography," J. Neurosurg. 49(5), 658-668 (1978).

4. K. R. Davis et al., "A review of the findings on computed cranial tomography following intravenous contrast media," Rev. Interam. Radiol. 2(1), 15-18 (1977).

5. I. Isherwood and B. R. Pullan, "CT of the spine. Applications and limitations," J. Comput. Assist. Tomogr. 1(3), 368 (1977).

6. M. Brant-Zawadzki, V. D. Burke, and R. B. Jeffrey, "CT in the evaluation of spine infection," Spine 8(4), 358-364 (1983).

7. A. L. McNamara et al., "Yield of image-guided needle biopsy for infectious discitis: a systematic review and meta-analysis," Am. J. Neuroradiol. 38(10), 2021-2027 (2017).

8. P. F. New et al., "Computerized axial tomography with the EMI scanner," Radiology 110(1), 109-123 (1974).

9. W. R. Scott et al., "Computerized axial tomography of intracerebral and intraventricular hemorrhage," Radiology 112(1), 73-80 (1974).

10. M. Brant-Zawadzki and R. B. Jeffrey Jr., "CT with image reformation for noninvasive screening of the carotid bifurcation: early experience," Am. J. Neuroradiol. 3(4), 395-400 (1982).

11. B. M. Brown, M. Brant-Zawadzki, and C. E. Cann, "Dynamic CT scanning of spinal column trauma," Am. J. Roentgenol. 139(6), 1177-1181 (1982).

12. S. Napel et al., "CT angiography with spiral CT and maximum intensity projection," Radiology 185(2), 607-610 (1992).

13. L. Axel, "Cerebral blood flow determination by rapid-sequence computed tomography: theoretical analysis," Radiology 137(3), 679-686 (1980).

14. D. Norman et al., "Dynamic computed tomography of the brain: techniques, data analysis, and applications," Am. J. Roentgenol. 136(4), 759-770 (1981).

15. M. Wintermark, "Perfusion CT imaging of acute ischemic brain injury with MSCT," in Multislice CT, M. F. Reiser et al., Eds., pp. 69-73, Springer, Berlin, Heidelberg (2004).

16. M. Koenig et al., "Perfusion $\mathrm{CT}$ of the brain: diagnostic approach for early detection of ischemic stroke," Radiology 209(1), 85-93 (1998).

17. C. Rincon-Guio, M. G. Ana, and D. C. Jose, "The role of computed tomography as a prognostic tool in traumatic brain trauma," Imaging Med. 9(6), 171-178 (1971).

18. M. N. Brant-Zawadzki et al., "High resolution CT with image reformation in maxillofacial pathology," Am. J. Roentgenol. 138(3), 477-483 (1982).

19. M. Schellenberg et al., "The impact of delayed time to first CT head in traumatic brain injury," Eur. J. Trauma Emerg. Surg. 47, 1511-1516. (2020).

20. N. Quartuccio et al., "The additional value of 18F-FDG PET and MRI in patients with Glioma: a review of the literature from 2015 to 2020," Diagnostics (Basel) 10(6), 357 (2020).

21. M. Unterrainer et al., "Recent advances of PET imaging in clinical radiation oncology," Radiat. Oncol. 15(1), 88 (2020). 
22. H. Shooli et al., "Theranostics in brain tumors," PET Clin. 16(3), 397-418 (2021).

23. W. N. Hanafee et al., "Computerized tomography scanning of the temporal bone," Ann. Otol. Rhinol. Laryngol. 88(5 Pt 1), 721-728 (1979).

24. A. A. Mancuso et al., "The role of computed tomography in the management of cancer of the larynx," Radiology 124(1), 243-244 (1977).

25. I. I. Kricheff et al., "Air-CT cisternography and canalography for small acoustic neuromas," Am. J. Neuroradiol. 1(1), 57-63 (1980).

26. J. L. Weissman and R. L. Carrau, “Puffed-cheek' CT improves evaluation of the oral cavity," Am. J. Neuroradiol. 22(4), 741-744 (2001).

27. US Burden of Disease Collaborators et al., "The state of US health, 1990-2016: burden of diseases, injuries, and risk factors among US states," JAMA 319(14), 1444-1472 (2018).

28. L. H. Kim et al., "Expenditures and health care utilization among adults with newly diagnosed low back and lower extremity pain," JAMA Network Open 2(5), e193676 (2019).

29. L. W. Roub and B. P. Drayer, "Spinal computed tomography: limitations and applications," Am. J. Roentgenol. 133(2), 267-273 (1979).

30. G. Di Chiro and D. Schellinger, "Computed tomography of spinal cord after lumbar intrathecal introduction of metrizamide (computer-assisted myelography)," Radiology 120(1), 101-104 (1976).

31. M. C. Mabray et al., "(18)F-sodium fluoride PET-CT hybrid imaging of the lumbar facet joints: tracer uptake and degree of correlation to CT-graded arthropathy," World J. Nucl. Med. 15(2), 85-90 (2016).

32. S. Usmani et al., "18F-sodium fluoride bone PET-CT in symptomatic lumbosacral transitional vertebra," Clin. Radiol. 75(8), 643.e1-643.e10 (2020).

33. S. Gamie and T. El-Maghraby, "The role of PET/CT in evaluation of Facet and Disc abnormalities in patients with low back pain using (18)F-fluoride," Nucl. Med. Rev. Cent. East. Eur. 11(1), 17-21 (2008).

34. C. R. Fitz and D. C. Harwood-Nash, "Computed tomography in hydrocephalus," J. Comput. Tomogr. 2(2), 91-108 (1978).

35. S. Hakim and R. D. Adams, "The special clinical problem of symptomatic hydrocephalus with normal cerebrospinal fluid pressure. Observations on cerebrospinal fluid hydrodynamics," J. Neurol. Sci. 2(4), 307-327 (1965).

36. B. P. Drayer, A. E. Rosenbaum, and H. B. Higman, "Cerebrospinal fluid imaging using serial metrizamide CT cisternography," Neuroradiology 13(1), 7-17 (1977).

37. W. I. Schievink et al., "Spontaneous spinal cerebrospinal fluid leaks and intracranial hypotension," J. Neurosurg. 84(4), 598-605 (1996).

38. R. A. Fishman and W. P. Dillon, "Dural enhancement and cerebral displacement secondary to intracranial hypotension," Neurology 43(3 Pt 1), 609-611 (1993).

39. P. G. Kranz et al., "Update on the diagnosis and treatment of spontaneous intracranial hypotension," Curr. Pain Headache Rep. 21(8), 37 (2017).

40. B. Mokri, D. G. Piepgras, and G. M. Miller, "Syndrome of orthostatic headaches and diffuse pachymeningeal gadolinium enhancement," Mayo Clin. Proc. 72(5), 400-413 (1997).

41. W. B. Oatway, A. L. Jones, and S. Holmes, Ionising Radiation Exposure of the UK Population, 2010 Review, Centre for Radiation, Chemical and Environmental Hazards, Public Health England (2016).

42. J. M. Meulepas et al., "Radiation exposure from pediatric CT scans and subsequent cancer risk in the Netherlands," J. Natl. Cancer Inst. 111(3), 256-263 (2019).

43. F. P. Probst, "Computer tomography of the skull and brain in evaluation of neuropediatric problems. Experiences and viewpoints. Part I," Diagn. Imaging 49(2), 61-76 (1980).

44. F. P. Probst, "Computer tomography of the skull and brain in evaluation of neuropediatric problems. Experiences and viewpoints. Part II," Diagn. Imaging 49(3), 113-122 (1980).

45. K. P. Hymel et al., "Comparison of intracranial computed tomographic (CT) findings in pediatric abusive and accidental head trauma," Pediatr. Radiol. 27(9), 743-747 (1997).

46. B. R. Foerster et al., "Neuroimaging evaluation of non-accidental head trauma with correlation to clinical outcomes: a review of 57 cases," J. Pediatr. 154(4), 573-577 (2009).

47. T. M. Shepherd et al., "Reducing patient radiation dose during CT-guided procedures: demonstration in spinal injections for pain," Am. J. Neuroradiol. 32(10), 1776-1782 (2011). 
48. V. N. Shah et al., "Long-term effectiveness of direct CT-guided aspiration and fenestration of symptomatic lumbar facet synovial cysts," Am. J. Neuroradiol. 39(1), 193-198 (2018).

49. T. J. Dietrich et al., "Fluoroscopy-guided versus CT-guided lumbar steroid injections: comparison of radiation exposure and outcomes," Radiology (290), 752-759 (2019).

William P. Dillon is the Elizabeth A. Guillaumin Professor of Radiology and Executive Vice Chair of Radiology at the University of California in San Francisco where he has worked since 1982. He is a past president of the American Society of Neuroradiology and the American Society of Head and Neck Radiology, and received the gold medal from both organizations. He has published over 350 articles and book chapters. 